\title{
Sustainability Code / Load Index for the Semi-quantitative Assessment of Analytical Methods in a Research Environment: Proof of Concept
}

\author{
Sergio Petrozzi", Chahan Yeretzian \\ Institute of Chemistry and Biological Chemistry, Zurich University of Applied Sciences ZHAW, Switzerland
}

Copyright (C) 2015 by authors, all rights reserved. Authors agree that this article remains permanently open access under the terms of the Creative Commons Attribution License 4.0 International License

\begin{abstract}
The aim of this project was to develop a general concept to assess the laboratory experiments in the instrumental analysis laboratory, at the Institute of Chemistry and Biological Chemistry (ICBC) at the Zurich University of Applied Sciences (ZHAW), in terms of their sustainability and, where possible, to improve the sustainability rating of the laboratory experiments. Hence, the primary objective was to implement an empirical assessment methodology, including the "Sustainability Code" that was composed of four parts and a "Sustainability Load Index". During the spring semester 2015, 105 different experimental projects were performed in the ICBC's analytical chemistry practical course. These were carried out on 18 different instrumental workspaces, encompassing 12 different analytical methodologies. For the development of the first and second parts of the Sustainability Code, information concerning the chemical substances (in any state- solid, liquid or gas) used and the energy consumption of the apparatus was recorded. The third and fourth parts contain (approximate) information on the use of consumables and the sustainability of the method with reference to the apparatus used. The Load Index (LI) consists of an aggregation (summary) of the direct causality indicators 'substances' and 'energy' into a single figure. The experiments were classified on a 3-level Load Index Scale into 'ideal' analysis methods (LI> 20), 'sustainable' methods of analysis (LI 20-200), and analysis methods 'with potential for optimization' (LI <200). The assessment criteria described here, specifically designed for an analytical process in a university environment, may also serve as a useful guide for semi-quantitative assessment of both familiar and new methods in analytical chemistry practical courses and in laboratory practice. It may also be useful in general research laboratory environments.
\end{abstract}

Keywords Green Analytical Chemistry, Sustainability Studies, Teaching Laboratory Classes, Green Metrics, Assessment Methods, Weighting, Sustainability Code, Load Index

\section{Introduction}

"About thirty of us had managed to surmount the harsh barrier of the first exams and had been admitted to the second year's Qualitative Analysis laboratory. We had entered that enormous, dark, smoky hall like someone who, coming into the House of the Lord, reflects on each of his steps... In this place, too, nobody wasted many words teaching us how to protect ourselves from acids, caustics, fires, and explosions; it appeared that the Institute's rough and ready morality counted on the process of natural selection to pick out those among us most qualified for physical and professional survival. There were few ventilation hoods; each student, following his text's prescriptions, in the course of systematic analysis, conscientiously let loose into the air a good dose of hydrochloric acid and ammonia, so that a dense, hoary mist of ammonium chloride stagnated permanently in the lab, depositing minute scintillating crystals on the window panes..."

This was how Primo Levi [1], an Italian chemist and writer, described a chemistry internship in 1939 in his book The Periodic Table.

Over time, the educational goal of student chemistry internships has not changed, but today the demands placed on sustainability in chemical education have altered dramatically [2-5]. The concept of sustainability refers (in very simple terms) to the environmentally sound use of resources. The question of sustainability used to be applied mainly to industrial chemical processes. Against the backdrop of today's fast-paced development of instrumental and chemical analysis techniques, it is not surprising that many conventional laboratory methods which are still included in a chemist's training need to be assessed and revised in terms of their environmental impact $[6,7]$.

In 1998 Paul Anastas and John Warner [8] published an 
integrated approach with 12 principles for sustainable chemistry under the title "Green Chemistry". The main objectives were not only to reduce materials causing pollution or replace them with less toxic compounds, but also to make increased use of renewable raw materials, select catalysts in preference to stoichiometric reagents, and minimize the generation of waste products.

Organic synthesis is the branch of chemistry that uses the most hazardous materials, while also contributing to their production. For this reason, scientific publications in the field of Green Chemistry have been dominated in recent decades by the optimization of organic processes with a focus on sustainability [9-12].

The need to quantify sustainability, for example in process comparisons, has resulted in different approaches to defining the parameters used for assessing environmental performance and applying them in assessment methods. However, these methods assess chemical processes mainly through maximizing the final product in terms of the total mass used, for example using factors such as Atom Economy [13], Effective Mass Yield [14], or Waste Generated, as in the Environmental Factor (E Factor) method [15].

These identifying metrics, which were designed for use in organic chemistry or biochemistry, cannot generally be used for classification in green analytical chemistry. The 2006 EcoScale, published by Aken et al. [16], describes a semi-quantitative assessment method for calculating the sustainability of processes in organic chemistry. It is based on the allocation of penalty points for deviation from pre-defined criteria for an 'ideal' synthesis. The sum of these relatively assigned penalty points shows the degree of sustainability of the process and allows a quick comparison of different procedures.

In recent years, interest in the implementation of the principles of Green Chemistry in analytical chemistry has grown dramatically and has resulted in numerous scientific publications [17-22].

Despite every effort to attain sustainability, each analytical method is always a compromise between ecological and economic viewpoints. Efforts to achieve more environmentally-friendly analytics include strategies such as the replacement of wet chemistry in sample preparation and treatment [23], the search for alternative solvents and reagents [24], direct analysis of samples without pretreatment [25], reduction of sample volume and miniaturization of apparatus [26], and automation [27].

In many cases, improvements in sustainability have been achieved through a substitution of the analytical methods used, for example the introduction of Supercritical Fluid Chromatography (SFC) in liquid chromatography [28-29] and the use of different apparatus, as in the introduction of Nano-Liquid Chromatography (nano LC) [30-31]. Another strategy to improve sustainability resulted in greatly modified processes, e.g. the use of miniaturized extraction techniques in sample preparation [32].

An analyte can be identified and quantified through a number of analytical methods, some of which could be replaced if the only goal were to improve sustainability. However, as the analytical methods used in practical training situations at universities are intended to give students the widest possible insight into various branches of instrumental analysis, replacement of the methods used is not normally desirable. This is especially because the existing experiments were primarily selected for educational reasons. Moreover, other aspects, such as quality of results, accuracy, precision, selectivity, detection limits and standardized procedures, are usually given extra weight in a training environment.

Following the example of the EcoScale, which was developed to assess organic chemistry procedures [16], Gałuska et al. [33] published an "Analytical Eco-Scale" for calculating the sustainability of analytical methods. In this calculation procedure penalty points were awarded based on previously defined environmental impact criteria.

In the present investigation two factors (the Sustainability Code and the Load Index) were introduced; these take into account the toxicity or hazard potential of the substances used for a given method as well as their quantities, the energy requirements of the method, and the type of waste disposal, in order to assess and compare the sustainability of analytical methods in a semi-quantitative way.

\section{The 12 Principles of Green Chemistry}

The concept of sustainable design in industrial production was concretized for the chemical industry at the beginning of the 1990s, inter alia in a program developed by the US Environmental Protection Agency (EPA). The contents and objectives of the concept can be summarized in 12 principles [8]:

\section{Prevention}

It is better to prevent waste than to treat or clean up waste after it has been created.

\section{Atom Economy}

Synthetic methods should be designed to maximize the incorporation of all materials used in the process into the final product.

\section{Less Hazardous Chemical Syntheses}

Wherever practicable, synthetic methods should be designed to use and generate substances that possess little or no toxicity to human health and the environment.

\section{Designing Safer Chemicals}

Chemical products should be designed to affect their desired function while minimizing their toxicity.

\section{Safer Solvents and Auxiliaries}

The use of auxiliary substances (e.g., solvents, separation agents, etc.) should be made unnecessary wherever possible and innocuous when used.

\section{Design for Energy Efficiency}

Energy requirements of chemical processes should be recognized for their environmental and economic impacts and should be minimized. If possible, synthetic methods 
should be conducted at ambient temperature and pressure.

\section{Use of Renewable Feedstocks}

A raw material or feedstock should be renewable rather than depleting whenever technically and economically practicable.

\section{Reduce Derivatives}

Unnecessary derivatization (use of blocking groups, protection/deprotection, temporary modification of physical/chemical processes) should be minimized or avoided if possible, because such steps require additional reagents and can generate waste.

\section{Catalysis}

Catalytic reagents (as selective as possible) are superior to stoichiometric reagents.

\section{Design for Degradation}

Chemical products should be designed so that at the end of their function they break down into innocuous degradation products and do not persist in the environment.

\section{Real-time Analysis for Pollution Prevention}

Analytical methodologies need to be further developed to allow for real-time, in-process monitoring and control prior to the formation of hazardous substances.

12. Inherently Safer Chemistry for Accident Prevention

Substances and the form of a substance used in a chemical process should be chosen to minimize the potential for chemical accidents, including releases, explosions, and fires.

\section{Analytical Practical Training}

In order to provide students with the broadest possible insight into modern analytics while taking a wide range of topics into account, a variety of analytical methods are used in the Analytical Chemistry Practical Basic Training Course at the ICBC (Table 1). Many different real samples are integrated into the experiments, e.g. pharmaceuticals, wastewater, and steels. Such experiments are designed to provide practical experience of the working techniques of an analytical chemist in terms of method development and validation. Nowadays (Figure 1), as in the past (Figure 2), the aim of the practical training is to enable students to select the correct analytical measurement methods for a particular analytical problem on the basis of literature studies, and to optimize and validate the method.

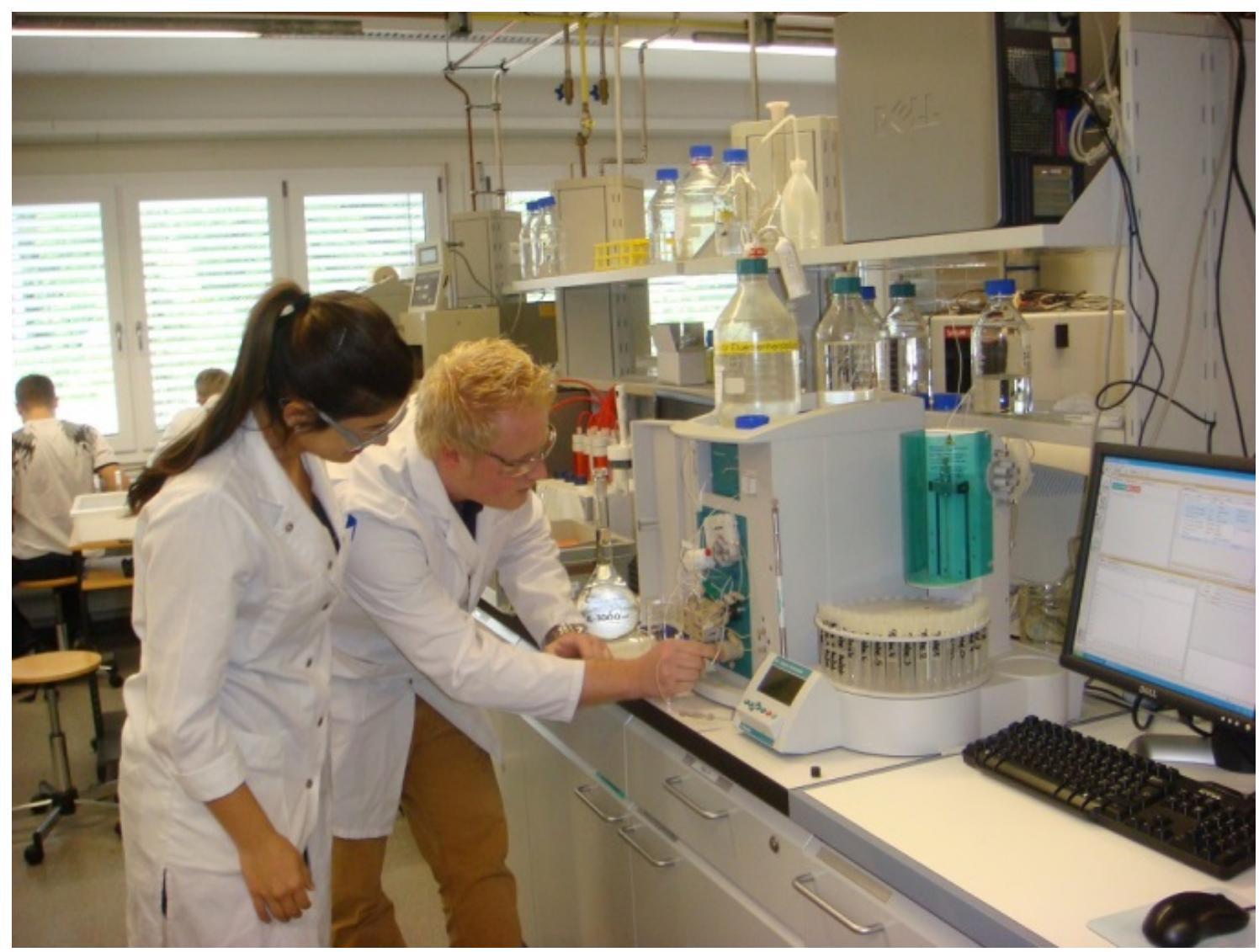

Figure 1. Practical Training (ZHAW, Wädenswil, 2015) 


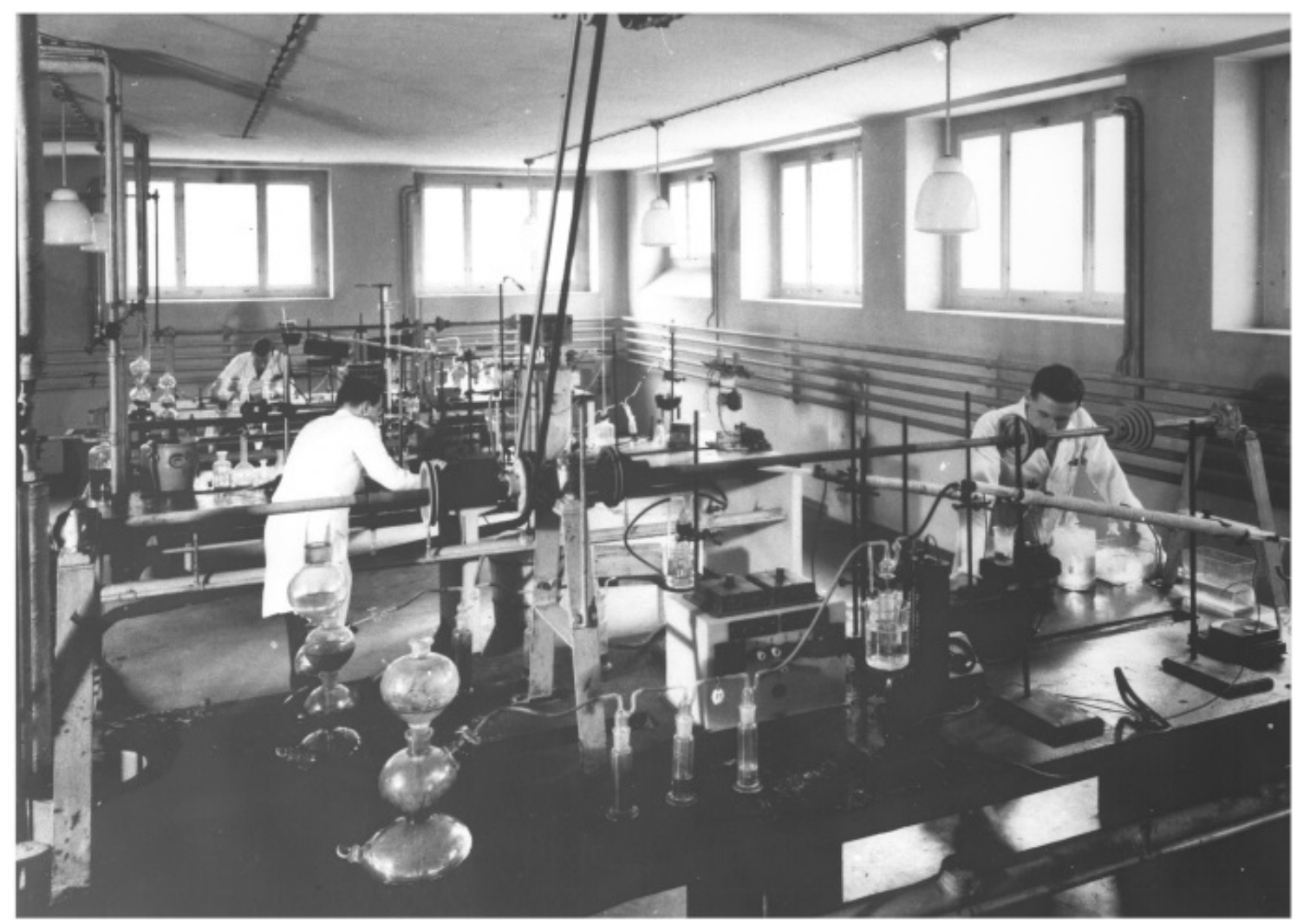

Figure 2. Practical Training Laboratory (Technikum Winterthur, 1948)

Three days in the laboratory are normally allowed for students to carry out a project. After this period, a report (part of the laboratory journal) is submitted, which is then assessed by the supervisors.

Table 1. Analytical Methods and Associated Tasks Used in the Practical Training Course at the ICBC

\begin{tabular}{c|c}
\hline Short Name of Experiment & Analytical Method \\
Definition of Project \\
\hline GC (1) & Gas Chromatography \\
GC (2) & Quantification of diesel oil in sand samples \\
Headspace Gas Chromatography & Quantification of ethanol in beer \\
HPLC (1,2) & High Performance Liquid Chromatography \\
IC & Quantification of drugs in analgesics \\
(1) & Ion Chromatography \\
(2) & Quantification of ions in beer \\
HPTLC & Quantification of ions in wine \\
UV-VIS & High-Performance Thin Layer Chromatography \\
Quantification of drugs in analgesics \\
Ultraviolet-Visible Spectroscopy
\end{tabular}




\section{Assessing the Analytical Process}

There are various possible approaches to improving the sustainability of each analytical process step (sampling, transport, sample preparation, analysis and disposal) in Green Chemistry. The assessment and optimization potential of the analytical methods investigated in the ICBC's practical training in analytics is based on a comparison with the previously existing experimental setup and thus results in a relative statement. In this context no practically-oriented 'absolute measure' of sustainability can be obtained, since reference values are not available. Furthermore, the Load Index of each method is based on a numerical weighting of materials and energy demand using subjective key figures which cannot be directly compared to each other because they are empirical.

Generally, however, it can be said that a sustainable method of analysis would have to meet the following criteria:

(1) The substances used do not present physical, environmental or health hazards.

(2) The quantities of materials used are kept to a minimum.

(3) The apparatus used for analysis (together with the peripheral devices) has low energy requirements.

(4) No (or very little) waste is generated.

(5) No (or little) sample preparation is required.

(6) Portable instruments are used to avoid transport and preservation.

(7) The method is inexpensive.

\subsection{Assessment Criteria}

The assessment criteria selected in this study are divided into three main categories:

\section{Semi-quantitative Causality Indicators}

With semi-quantitative causality indicators, such as the safety aspect in terms of substances and energy, there is a direct causal relationship to sustainability.

\section{Qualitative Causality Indicators}

For qualitative causality indicators, the factors can only be quantified in terms of subjectively determined potentials. Examples are additivity of hazards, recycling/disposal (of materials, devices), and the lifespan of apparatus. Although consumables and the weights of devices can be objectively quantified, their contribution to pollution can only be subjectively indicated.

\section{Holistic Perspective}

The consideration of all indicators, including the purchase price of substances and devices and the labor necessary to operate the equipment, is beyond the scope of this study. For this reason, the following additional factors have not been taken into account:

- Origin of raw materials (working conditions for production, local environmental regulations etc.)

- Resource consumption and environmental impact during manufacture
- Energy demand during the production and disposal of materials and devices/apparatus.

\subsection{The Sustainability Code and Load Index}

The individual steps of the analytical process - sampling, preservation and transportation - were not taken into account in this study, since they do not play an essential role in practical training. During the ICBC Practical Training Course in the spring semester 2015, all substances used and the technical specifications of the devices/apparatus used for a particular experiment were listed and used for assessment purposes. The data collected relate to a trial period of 3 days (project duration) with a presence time of 8 hours per day; the equipment was constantly under power throughout the day.

\subsection{Assessment Procedure Used}

The assessments conducted during this study provided figures based on a ZHAW-specific period of practical training, with an average total sample throughput of 35-40 analyses. The method presented here for determining the Sustainability Code or Load Index comprises the following steps:

1. Definition of the different phases of the analytical process

The following were the essential sub-phases selected for the assessment process:
A. Sample preparation
B. Analysis
C. Disposal

2. Assignment of a Load Index to the Substances with Respect to Safety

In accordance with the basic hazard potential (BHP) described in the Basic Hazard Class, each of the substances used in a sub-step was assigned an extended hazard potential value (EHP) (Tables 2 and 3). The 'most hazardous' component (the one with the highest hazard number) was established (BHP x EHP) and considered on its own as representing all the materials used for the assessment. A qualitative graphical representation of the potential hazards of all substances was shown in the form of a spider diagram. Each hazard listed in the GHS (Globally Harmonized System of Classification and Labelling of Chemicals) was shown as an axis (see point $\mathrm{E}$ of the weighting procedure, Section 5.1). In the next step various Potentiation Factors were linked to the 'most hazardous' component (absolute amounts of substances, number of hazards, recycling/disposal), and the Load Index 'Substances' (LI) was thereby determined.

$$
\begin{array}{lll}
\multicolumn{2}{c}{\text { LI'Substances' }=\left((\mathrm{BHP} * \mathrm{EHP}) * P_{Q} * P_{P I C} * P_{R / D}\right) / 10} \\
\text { BHP } & \text { Basic Hazard Potential } & \text { (Table 2) } \\
\text { EHP } & \text { Extended Hazard Potential } & \text { (Table 3) } \\
\mathrm{P}_{\mathrm{Q}} & \text { Potentiation Factor 'Quantity' } & \text { (Table 4) } \\
\mathrm{P}_{\mathrm{PIC}} & \text { Potentiation Factor 'Pictograms' } & \text { (Table 5) } \\
\mathrm{P}_{\mathrm{R} / \mathrm{D}} & \text { Potentiation Factor 'Recycling/Disposal' } & \text { (Table 6) }
\end{array}
$$

The divisor 10 was subjectively introduced to obtain Load 
Indices with similar figures.

\section{Assignment of the Load Index 'Consumables' (LI C)}

The Load Index 'Consumables' corresponds to the quantity of consumables used (see Section 3.8).

\section{Load Index 'Energy Demand' (LI E)}

The Load Index 'Energy Demand' corresponds to the total consumption of energy by all devices and apparatus (see Section 4.9).

\section{Assignment of the Load Index 'Devices and Apparatus' (LI D/A)}

To calculate the load caused by devices and apparatus, the Potentiation Factors 'Weight' and 'Lifespan' were taken into account:

$$
\text { LI D/A }=F_{W} * P_{E D}
$$

$$
\begin{array}{lll}
F_{W} & \text { Factor 'Weight' } & \text { (Table 8) } \\
P_{E D} & \text { Potentiation Factor 'Lifespan' (Table 9) }
\end{array}
$$

\section{Qualitative Representation of the Methods}

A figurative and qualitative representation of the entire method was created on the basis of the information obtained.

\section{Calculation of the Sustainability Code (Overall Method)}

The first part of the proposed Sustainability Code contains the Load Index for the substances used (S); the load caused by the consumables $(\mathrm{C})$ can be seen in the second part, and in the third part the energy demand over the period of the experiment (E) is shown. The fourth part of the Code contains information on device-related sustainability (D/A), related to weight and lifespan (see Section 4.1).
Sustainability code: S-C-E-D/A
Reference parameters:
$\mathrm{S}$ (Factor)
$\mathrm{E}(\mathrm{kW} / \mathrm{h})$
C (Quantity)
$\mathrm{D} / \mathrm{A}\left(\mathrm{kg} * P_{E D}\right)$

8. Load Index (LI) for a Given Experiment

The Load Index consists of a compression of the partial indices Substances and Energy into a single key figure, as these two values can be determined semi-quantitatively. The higher the values of the indices, the lower the sustainability.

$$
\mathrm{LI}_{\text {Experiment }}=\mathrm{LI}_{\text {Substances }}+\mathrm{LI}_{\text {Energy }}
$$

\subsection{The Safety Aspect 'Substances' (S)}

The GHS system was used as the basis for weighting the substances used in this process. The parameters were weighted and (relative) Potentiation Factors were allocated: these were not always based on clearly defined values and parameters, but sometimes on experience and intuition. The various hazard types of the materials used were divided into the respective GHS hazard classes. Each of these hazard classes was assigned a Basic Hazard Potential (BHP) (Table 2 ), whereby a hazard to human health was assigned the highest potential. The substances selected were those which need to be handled by students in a learning environment, and special attention was therefore paid to human hazard and accident prevention. Within the hazard classes a further division was made into subcategories depending on extent/severity: Extended Hazard Potentials (EHP) (Table 3) were assigned to each hazard class for this purpose.

Table 2. The Basic Hazard Potentials (BHP) Associated with Each Hazard Class

\begin{tabular}{c|c|cc}
\hline Hazard Class & BHP & The likelihood of an adverse event is weighed against the potential impacts. \\
\hline $\begin{array}{c}\text { GHS01 } \\
\text { Unstable }\end{array}$ & n.r & Likelihood of an adverse event: not relevant for practical training. These materials are not used in the practical \\
training at the ICBC.
\end{tabular}


Table 3. Basic and Extended Hazard Potentials

\begin{tabular}{|c|c|c|c|c|}
\hline $\begin{array}{l}\text { GHS Pictogram, } \\
\text { Basic Hazard } \\
\text { Potential (GGP) }\end{array}$ & Code & $\begin{array}{l}\text { Hazard Statement Based on } \\
\text { EG No. 1272/2008 }\end{array}$ & $\begin{array}{c}\text { Basic } \\
\text { Hazard } \\
\text { Potential } \\
\text { (BHP) } \\
\end{array}$ & $\begin{array}{l}\text { Extended Hazard } \\
\text { Potential } \\
\text { (EGP) }\end{array}$ \\
\hline GHS 01 & $\begin{array}{l}\text { H200 } \\
\text { H201 } \\
\text { H202 } \\
\text { H203 } \\
\text { H204 } \\
\text { H240 } \\
\text { H241 }\end{array}$ & $\begin{array}{l}\text { Unstable explosives } \\
\text { Explosive; mass explosion hazard } \\
\text { Explosive, severe projection hazard } \\
\text { Explosive; fire, blast or projection hazard } \\
\text { Fire or projection hazard } \\
\text { Heating may cause an explosion. } \\
\text { Heating may cause a fire or explosion. }\end{array}$ & & $\begin{array}{l}9 \\
6 \\
6 \\
6 \\
6 \\
3 \\
3\end{array}$ \\
\hline GHS 02 & $\begin{array}{l}\text { H220 } \\
\text { H221 } \\
\text { H222 } \\
\text { H223 } \\
\text { H224 } \\
\text { H225 } \\
\text { H226 } \\
\text { H228 } \\
\text { H241 } \\
\text { H242 } \\
\text { H250 } \\
\text { H251 } \\
\text { H252 } \\
\text { H260 } \\
\text { H261 } \\
\end{array}$ & $\begin{array}{l}\text { Extremely flammable gas } \\
\text { Flammable gas } \\
\text { Extremely flammable aerosol } \\
\text { Flammable aerosol } \\
\text { Extremely flammable liquid and vapor } \\
\text { Highly flammable liquid and vapor } \\
\text { Flammable liquid and vapor } \\
\text { Flammable solid } \\
\text { Heating may cause a fire or explosion. } \\
\text { Heating may cause a fire. } \\
\text { Catches fire spontaneously if exposed to air. } \\
\text { Self-heating: may catch fire. } \\
\text { Self-heating in large quantities; may catch fire. } \\
\text { In contact with water releases flammable gases which may ignite spontaneously. } \\
\text { In contact with water releases flammable gases. }\end{array}$ & 4 & $\begin{array}{l}9 \\
3 \\
9 \\
3 \\
9 \\
6 \\
3 \\
3 \\
5 \\
3 \\
3 \\
9 \\
9 \\
3 \\
9 \\
3 \\
3\end{array}$ \\
\hline & $\begin{array}{l}\mathrm{H} 270 \\
\mathrm{H} 271 \\
\mathrm{H} 272\end{array}$ & $\begin{array}{l}\text { May cause or intensify fire; oxidizer. } \\
\text { May cause fire or explosion; strong oxidizer. } \\
\text { May intensify fire; oxidizer. }\end{array}$ & 5 & $\begin{array}{l}5 \\
7 \\
3\end{array}$ \\
\hline & $\begin{array}{l}\mathrm{H} 280 \\
\mathrm{H} 282\end{array}$ & $\begin{array}{l}\text { Contains gas under pressure; may explode if heated. } \\
\text { Contains refrigerated gas; may cause cryogenic burns or injury. }\end{array}$ & 2 & $\begin{array}{l}1 \\
3\end{array}$ \\
\hline & $\begin{array}{l}\text { H290 } \\
\text { H314 } \\
\text { H318 }\end{array}$ & $\begin{array}{l}\text { May be corrosive to metals. } \\
\text { Causes severe skin burns and eye damage. } \\
\text { Causes serious eye damage. }\end{array}$ & 12 & $\begin{array}{l}3 \\
9 \\
9\end{array}$ \\
\hline & $\begin{array}{l}\text { H300 } \\
\text { H301 } \\
\text { H310 } \\
\text { H311 } \\
\text { H330 } \\
\text { H331 }\end{array}$ & $\begin{array}{l}\text { Fatal if swallowed } \\
\text { Toxic if swallowed } \\
\text { Fatal in contact with skin } \\
\text { Toxic in contact with skin } \\
\text { Fatal if inhaled } \\
\text { Toxic if inhaled }\end{array}$ & 16 & $\begin{array}{l}9 \\
7 \\
9 \\
7 \\
9 \\
7\end{array}$ \\
\hline GHS 07 & $\begin{array}{l}\text { H302 } \\
\text { H312 } \\
\text { H315 } \\
\text { H317 } \\
\text { H319 } \\
\text { H332 } \\
\text { H335 } \\
\text { H336 } \\
\end{array}$ & $\begin{array}{l}\text { Harmful if swallowed } \\
\text { Harmful in contact with skin } \\
\text { Causes skin irritation. } \\
\text { May cause an allergic skin reaction. } \\
\text { Causes serious eye irritation. } \\
\text { Harmful if inhaled } \\
\text { May cause respiratory irritation. } \\
\text { May cause drowsiness or dizziness. }\end{array}$ & 6 & $\begin{array}{l}3 \\
3 \\
2 \\
2 \\
7 \\
3 \\
4 \\
3\end{array}$ \\
\hline
\end{tabular}




\begin{tabular}{|c|c|c|c|c|}
\hline GHS 08 & $\begin{array}{l}\text { H304 } \\
\text { H334 } \\
\text { H340 } \\
\text { H341 } \\
\text { H350 } \\
\text { H351 } \\
\text { H360 } \\
\text { H361 } \\
\text { H370 } \\
\text { H371 } \\
\text { H372 } \\
\text { H373 }\end{array}$ & $\begin{array}{l}\text { May be fatal if swallowed and enters airways. } \\
\text { May cause allergy or asthma symptoms or breathing difficulties if inhaled. } \\
\text { May cause genetic defects. } \\
\text { May cause genetic defects. } \\
\text { May cause cancer. } \\
\text { Suspected of causing cancer. } \\
\text { May damage fertility or the unborn child } \\
\text { Suspected of damaging fertility or the unborn child. } \\
\text { Causes damage to organs. } \\
\text { May cause damage to organs. } \\
\text { Causes damage to organs through prolonged or repeated exposure } \\
\text { May cause damage to organs through prolonged or repeated exposure. }\end{array}$ & $\begin{array}{c}8 \\
\text { H304 } 16\end{array}$ & $\begin{array}{l}\mathbf{9} \\
\mathbf{5} \\
\mathbf{9} \\
7 \\
\mathbf{9} \\
7 \\
\mathbf{9} \\
7 \\
\mathbf{9} \\
\mathbf{7} \\
\mathbf{6} \\
\mathbf{5}\end{array}$ \\
\hline & $\begin{array}{l}\text { H400 } \\
\text { H410 } \\
\text { H411 } \\
\text { H412 } \\
\text { H413 }\end{array}$ & $\begin{array}{l}\text { Very toxic to aquatic life } \\
\text { Very toxic to aquatic life with long lasting effects } \\
\text { Toxic to aquatic life with long lasting effects } \\
\text { Harmful to aquatic life with long lasting effects } \\
\text { May cause long lasting harmful effects to aquatic life }\end{array}$ & 3 & $\begin{array}{l}9 \\
9 \\
6 \\
3 \\
2\end{array}$ \\
\hline
\end{tabular}

\subsection{Quantity of Substances Used}

Since the influence of hazardous substances depends on their quantity, the absolute quantity of materials used was weighted with a Potentiation Factor.

Table 4. Potentiation Factor Assigned to the Quantity Used

\begin{tabular}{c|c}
\hline Mass of Substances $\left(\mathbf{g}, \mathbf{m l}, \mathbf{L}_{\text {Gas }}\right)$ & Potentiation Factor \\
\hline$<0.2$ & 1 \\
$>0.2-1$ & 1.5 \\
$>1-5$ & 2 \\
$>5-10$ & 3.5 \\
$>10-50$ & 4.5 \\
$>50-100$ & 7 \\
$>100-300$ & 15 \\
$>300-600$ & 20 \\
$>600-1000$ & 35 \\
\hline
\end{tabular}

It should be emphasized that in contrast to multi-component mixtures in which the danger and toxicity of individual substances could behave synergistically, in this study only the most dangerous classified component was considered for assessment, whereby the mass and not the concentration was decisive. Quantities below $200 \mathrm{mg}$ were not provided with a Potentiation Factor, or this was set to 1 (Table 4).

The factors introduced here were assigned to the quantities on a subjective basis. It was found that the maximum quantity of substances used during the practical training in the spring semester 2015 was approx. $1000 \mathrm{~g}$. This value was set as a maximum on the rating scale, the amount of $0.2 \mathrm{~g}$ was set as a threshold value, and the intervals in the scales of material mass and potentiation factors were selected empirically to achieve a meaningful statement of the hazards involved.

\subsection{Additivity of Hazards}

As individual substances may cause more than one hazard, any additional hazard (= pictogram) was also provided with a Potentiation Factor (Table 5). The Potentiation Factors were assigned subjectively (Table 5). For example, the Potentiation Factor for 2 pictograms results from $(2-1)=1$, which is added as a decimal to $1(1+0.1)$, enhancing the hazard by $10 \%$.

Table 5. Potentiation Factors when a Substance Presents Multiple Hazards

\begin{tabular}{c|c}
\hline Number of Pictograms & Potentiation Factor \\
\hline 1 & 1 \\
2 & 1.1 \\
3 & 1.2 \\
4 & 1.3 \\
5 & 1.4 \\
\hline
\end{tabular}

\subsection{Waste and Recycling/Disposal}

At present, (almost) the only type of disposal at the ICBC is external. This step is also given a Potentiation Factor (Table 6). No used materials are reprocessed and reused. In accordance with the waste handling methods described in the ICBC's Practical Training Guidelines, waste is separated before being collected by an external company and disposed of appropriately.

Table 6. Type of Disposal with Corresponding Potentiation Factor

\begin{tabular}{c|c}
\hline Type & Potentiation Factor \\
\hline Recycling & 0.2 \\
Sewerage & 1.0 \\
Neutralization & 1.1 \\
External disposal & 1.2 \\
\hline
\end{tabular}

\subsection{Consumables (C)}

Consumables in analytics primarily comprise filters, syringes, glass pipettes and rubber gloves. For filtration of small volume samples prior to chromatographic analysis (HPLC, GC or IC), membrane syringe filters are widely used, because particles must be avoided in the solutions to be analyzed. To carry out the analysis, the samples for analysis are introduced into vials, which are closed with screw caps, including septa. Unfortunately, no information on sustainability is available for the materials used at this stage, but the production of clean glass vessels can certainly be regarded as energy-intensive. One-way materials are disposed of as solid hazardous waste. The Load Index 'Consumables' corresponds to the quantity of the materials 
used, without taking their composition or type into account.

\subsection{Energy Demand (E)}

Energy analysis is a very important part of the life cycle inventory. Environmental problems are often linked to the sourcing of energy and the associated energy carriers. For this reason, the production of materials for analysis is linked to energy provision and energy requirements. The availability of resources (especially the fossil resources oil, natural gas and, to a lesser extent, coal) is limited. As can be seen from Table 7, the energy requirement of each individual device was totaled and the Sustainability Index for Energy (E) was derived from the resulting sum.

Table 7. Load Index representing Total Energy Demand

\begin{tabular}{c|c}
\hline Output $(\mathrm{kW} / \mathrm{h})$ & Load Index $(\mathrm{E})(\mathrm{kW} / \mathrm{h}$ total $)$ \\
\hline e.g. Device X 80 & $(80+20)$ \\
Peripheral device Y 20 & 100 \\
\hline
\end{tabular}

\subsection{Devices and Apparatus}

Devices and apparatus, and their composition, are a critical factor in the sustainability-oriented assessment of analytical methods. To account for environmental impacts that already arose in the production of devices and apparatus, several suppliers of the devices used in the lab were asked for specific data relating to their production. However, it was not possible to obtain data on the production conditions in this way. Either no surveys had been carried out or the relevant information was classified as confidential.

Assessment of apparatus was therefore carried out on the basis of energy demand and the lifespan of the apparatus. The information on the energy demand of devices mostly stems from user manuals. Swiss companies gave an assurance that they worked together with local suppliers when procuring materials and that their employees were mainly recruited locally.

\subsection{Weight of Apparatus}

The weight of the final product is a more frequently used reference unit for analytical instruments (in contrast to services). Information on weight gives a very rough idea of the resource input required to produce an analytical instrument. Weight was only used in this study as a representative value on which the assessment of sustainability in the production phase was based. Neither the composition of the individual components of the apparatus (e.g. iron, aluminum and copper) nor the use of fossil energy resources in the production or disposal processes was considered (Table 8).

Table 8. Weight as an Assessment Parameter

\begin{tabular}{c|c}
\hline Weight $(\mathrm{kg})$ & Factor Overall Weight \\
\hline $\begin{array}{c}\text { e.g. Device X 100 } \\
\text { Peripheral device Y 20 }\end{array}$ & $(80+20)=100$ \\
\hline
\end{tabular}

One possible approach to saving resources could be the re-use of analytical instruments. Operational and valuable components could be reused or recycled as part of the general overhaul of another device. No data is available about final disposal or possible recycling of the analytical devices used in the ICBC's Practical Training Course. An accurate and complete Life Cycle Analysis (LCA) of all devices would also be beyond the scope of this investigation and was therefore not carried out.

\subsection{Lifespan of the Apparatus}

The lifespan of a device has a definite impact on its environmental impact. Quality, hygiene and regular maintenance can greatly extend the life of equipment, thus contributing to resource conservation. It should be mentioned that most devices are very durable products and many spare parts are available for 10 years after termination of production. Devices may therefore be in operation for long periods, which is an argument in favor of a sustainability strategy and against disposable products. However, a major disadvantage of the long lifetime of a device, from the perspective of the ICBC's Practical Training Course, is the rapid progress of technological development, which can very quickly make an instrument obsolete and necessitate the replacement of outdated devices.

Table 9. Lifespan as an Assessment Parameter

\begin{tabular}{c|c}
\hline Use* (Years) & Potentiation Factor \\
\hline Over 20 & 1.0 \\
$10-20$ & 1.1 \\
Less than 10 & 1.2 \\
\hline
\end{tabular}

* From experience or expected lifetime

The environmental effects of maintenance and repairs which originate from the production of spare parts and of transport processes are certainly less pronounced than the consumption of resources and energy involved in the production and disposal of an entire new unit. Social sustainability factors also play a role (employment as a means of securing livelihood, preservation of social productive potential), but these cannot be clearly defined because of the subjectivity involved.

\section{Results}

While all laboratory experiments of the analytical chemistry lab courses of the ICBC were analyzed and assessed, according to the sustainability criteria outlined above, only two examples are discussed here: HPLC and AES. Sustainability assessments of all other experiments (Table 1) can be requested from the authors.

\subsection{Description of the Assessment Process}

When laboratory experiments are performed, various aspects of analytical methodology need to be taken into account and various tasks accomplished: these are grouped together under the analytical questions. After this the measurement method, apparatus and the associated peripherals are described. 
To calculate a Sustainability Code, Hazard Potentials then need to be assigned to the substances used (under A). These Hazard Potentials give a picture of the materials' ecological footprint and their impact in terms of sustainability (under $\mathbf{C}$ and D). For each hazard symbol (GHSOx) only the material with the highest Extended Hazard Potential is considered. The Hazard Potentials of these substances are then qualitatively summarized (under $\mathbf{E}$ ). In addition, any Potentiation Factors must be included in the calculation, such as the quantities of substances used (F), multiplicity of hazards (under $\mathbf{G}$ ), recycling aspects (under $\mathbf{H}$ ), and the consumables required (under I). Moreover, the apparatus (under B) is weighted according to the aspects of energy (under $\mathbf{J}$ ), weight of the apparatus as a measure of resource consumption in its production (under $\mathbf{K}$ ), and lifetime (under L). Subsequently, the Sustainability Code and Load Index are summarized under $\mathbf{M}$.

\subsection{HPLC Experiment}

\section{Analytical Problem}

Quantitative determination of paracetamol, caffeine and propyphenazone in pain relief tablets and validation of the method

A. Substances Used

\begin{tabular}{cc}
\hline Substance & CAS Number \\
\hline Caffeine & $58-08-2$ \\
\hline Paracetamol & $103-90-2$ \\
\hline Propyphenazone & $479-92-5$ \\
\hline Methanol & $67-56-1$ \\
\hline
\end{tabular}

B. Devices/Apparatus Used

\begin{tabular}{c}
\hline Main Devices \\
\hline Autosampler \\
\hline Diode Array Detector \\
\hline Degasser \\
\hline Binary pump \\
\hline Column compartment \\
\hline Fluorescence detector \\
\hline Computer
\end{tabular}

C. Hazard of the Substances Used

\begin{tabular}{ccc}
\hline Substance & Hazard & BHP \\
\hline \multirow{2}{*}{ Caffeine } & GHS07 & 3 \\
\hline \multirow{2}{*}{ Paracetamol } & H302 & \\
& H302 & 3 \\
& H315 & 2 \\
Propyphenazone & H319 & 6 \\
& H335 & 4 \\
\hline \multirow{2}{*}{ Methanol } & GHS07 & 3 \\
\hline & GHS02 & \\
& H225 & 6 \\
& GHS06 & 7 \\
& H301 & 7 \\
& H311 & 7 \\
& H331 & 9 \\
\hline
\end{tabular}

BHP: Basic Hazard Potential
D. Determination of the Hazard Potentials

\begin{tabular}{ccccc}
\hline HC & BHP & EHP & Hazard Number & Substance \\
\hline GHS02 & 4 & 6 & 24 & \\
\hline GHS03 & 5 & - & - & \\
\hline GHS04 & 2 & - & - & \\
\hline GHS05 & 12 & - & & \\
\hline GHS06 & 16 & 7 & 112 & \\
\hline GHS07 & 6 & 6 & 36 & \\
\hline GHS08 & 8 & 9 & 72 & \\
\hline GHS09 & 3 & - & & \\
\hline
\end{tabular}

HC: Hazard Class; BHP: Basic Hazard Potential; EHP: Extended Hazard Potential

E. Hazard Potentials

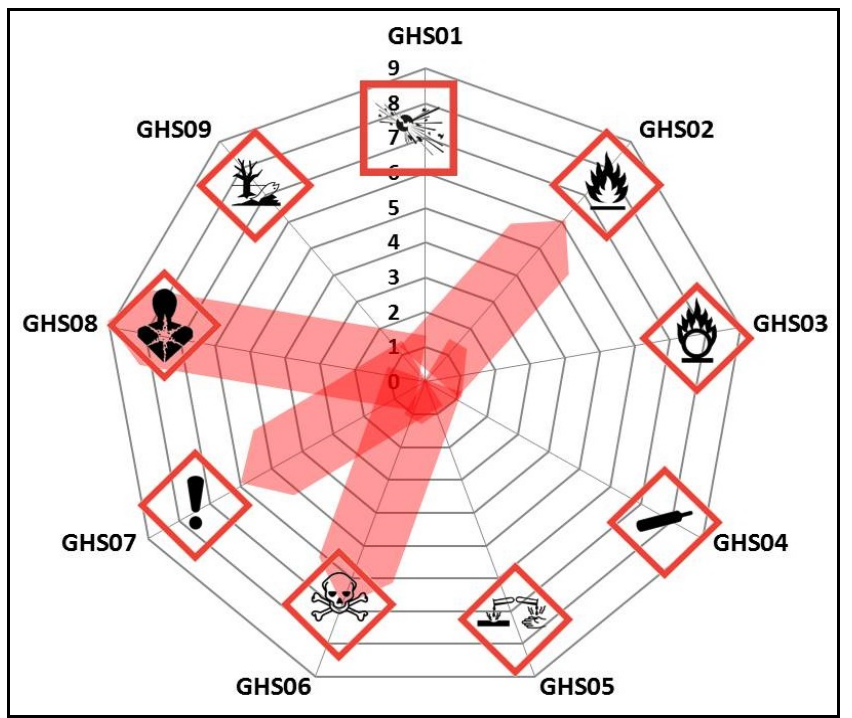

Figure 3. Qualitative Representation of the Substances Used in the HPLC Experiment

F. Substances Used (Absolute Quantities)

\begin{tabular}{ccc}
\hline Material & Quantity & Potentiation Factor \\
\hline Caffeine & $0.05 \mathrm{~g}$ & \\
Paracetamol & $0.1 \mathrm{~g}$ & \\
Propyphenazone & $0.1 \mathrm{~g}$ & \\
\hline Methanol & $\mathbf{8 5 6} \mathbf{~ m l}$ & $\mathbf{3 5}$ \\
\hline
\end{tabular}

G. Number of Hazards

\begin{tabular}{cc}
\hline Number of Pictograms & Potentiation Factor \\
\hline 3 & 1.2 \\
\hline
\end{tabular}

H. Recycling/Disposal

\begin{tabular}{cc}
\hline Type & Potentiation Factor \\
\hline External & 1.2 \\
\hline I. Consumables & \\
\hline Article & Quantity \\
\hline Glass vials & 30 \\
1-way syringes & 30 \\
1-way filters & 30 \\
\hline Load Index (C) & $\mathbf{9 0}$ \\
\hline
\end{tabular}


J. Energy Demand of Devices/Apparatus

\begin{tabular}{cc}
\hline Components & Energy Demand (kWh) \\
\hline Autosampler & 7.2 \\
\hline DAD & 3.84 \\
\hline Degasser & 0.7 \\
\hline Binary pump SL & 3.84 \\
\hline Column compartment & 7.7 \\
\hline Fluorescence detector & 4.3 \\
\hline Computer & 4.8 \\
\hline Load Index (E) & $\mathbf{3 2 . 4}$ \\
\hline
\end{tabular}

K. Weight of Devices/Apparatus

\begin{tabular}{cc}
\hline Components & Mass (kg) \\
\hline Autosampler & 14.2 \\
\hline DAD & 11.5 \\
\hline Degasser & 7 \\
\hline Binary pump SL & 15.5 \\
\hline Column compartment & 10.2 \\
\hline Fluorescence detector & 11.5 \\
\hline Computer & 21 \\
\hline Autosampler & 14.2 \\
\hline Factor Weight & $\mathbf{9 1}$
\end{tabular}

L. Lifetime of Devices/Apparatus

\begin{tabular}{cc}
\hline Use & Potentiation Factor \\
\hline Over 10 years & 1.1 \\
\hline
\end{tabular}

M. Sustainability Code \& Load Index Calculation

\begin{tabular}{cc}
\hline Substances & \\
\hline Basic Hazard Potential (BHP) & 16 \\
\hline Extended Hazard Potential (EHP) & 7 \\
\hline Hazard Number & $\mathbf{1 1 2}$ \\
\hline Potentiation Factor 'Quantity' & 35 \\
\hline Potentiation Factor 'Pictograms' & 1.2 \\
\hline Potentiation Factor 'Recycling/Disposal (R\&E)' & 1.2 \\
\hline Load Index (S) & $\mathbf{5 6 4}$ \\
\hline Consumables & $\mathbf{9 0}$ \\
\hline Load Index (C) \\
\hline Energy Demand \\
\hline Load Index (E) \\
\hline \\
\hline Devices/Apparatus \\
\hline Factor 'Weight' \\
\hline Potentiation Factor 'Lifespan' \\
\hline Load Index (D/A) \\
\hline
\end{tabular}

The Sustainability Code of the HPLC experiment was: S-564-C-90-E-32-D/A-100, while the Load Index was 596.

\subsection{AES Experiment}

\section{Analytical Problem}

Determination of sodium content in mineral water with external calibration, sodium analysis with the spike method and validation of the method

A. Substances Used

\begin{tabular}{cc}
\hline Substance & CAS Number \\
\hline $\mathrm{KCl}^{*}$ & $7447-40-7$ \\
\hline $\mathrm{NaCl}^{*}$ & $7467-14-5$ \\
\hline $\mathrm{CaCl} 2^{* *}$ & $10035-04-8$ \\
\hline Acetylene & $74-86-2$ \\
\hline
\end{tabular}

* Not taken into account as no hazardous materials as listed in the GHS

** Not taken into account as below the minimum quantity of $200 \mathrm{mg}$

B. Devices/Apparatus Used

\begin{tabular}{c}
\hline Main Devices \\
\hline Atom absorption/emission spectrometer \\
Computer \\
\hline
\end{tabular}

C. Degree of Hazard of the Substances Used

\begin{tabular}{ccc}
\hline Substance & Hazard & BHP Basic Hazard Potential \\
\hline \multirow{3}{*}{ Acetylene } & GHS03 & 5 \\
& H270 & \\
& GHS04 & 1 \\
\hline
\end{tabular}

D. Calculation of the Hazard Potentials

\begin{tabular}{ccccc}
\hline HC & BHP & EHP & Hazard Number & Substance \\
\hline GHS02 & 4 & - & & \\
\hline GHS03 & 5 & 5 & 25 & Acetylene \\
\hline GHS04 & 2 & 1 & 2 & \\
\hline GHS05 & 12 & - & & \\
\hline GHS06 & 16 & - & & \\
\hline GHS07 & 6 & - & & \\
\hline GHS08 & 8 & - & & \\
\hline GHS09 & 3 & - & & \\
\hline
\end{tabular}

HC: Hazard Class

BHP: Basic Hazard Potential

EHP: Extended Hazard Potential

E. Hazard Potentials

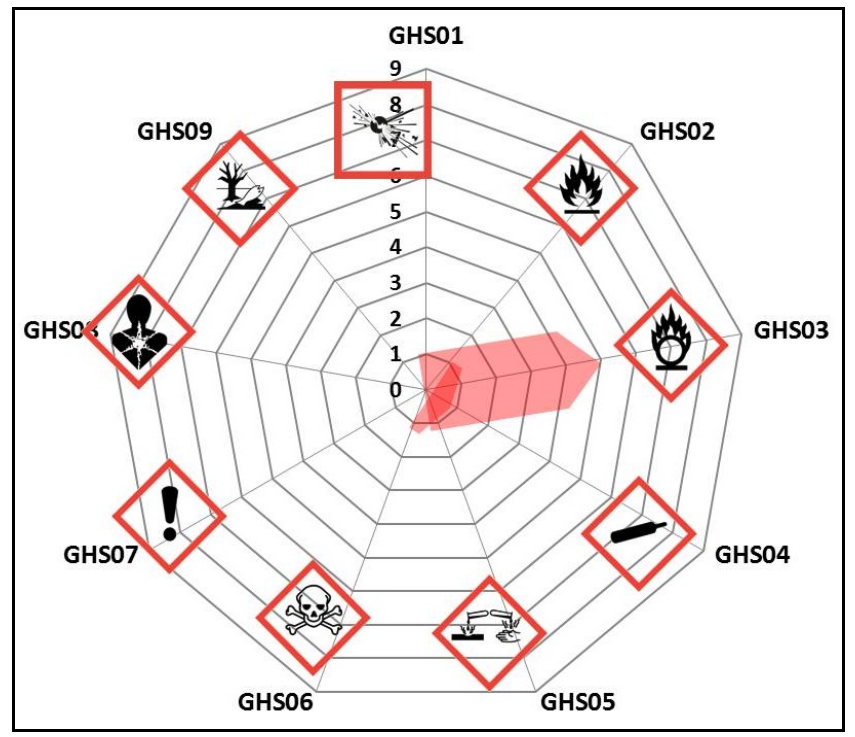

Figure 4. Qualitative Representation of the Substances Used for the AES Experiment 
F. Substances Used (Absolute Quantities)

\begin{tabular}{ccc}
\hline Substance & Quantity & Potentiation Factor \\
\hline Acetylene & 2001 & 15 \\
\hline
\end{tabular}

G. Number of Hazards

\begin{tabular}{cc}
\hline Number of Pictograms & Potentiation Factor \\
\hline 2 & 1.1 \\
\hline
\end{tabular}

H. Recycling/Disposal

\begin{tabular}{cc}
\hline Type & Potentiation Factor \\
\hline External & 1.2 \\
\hline
\end{tabular}

I. Consumables

\begin{tabular}{cc}
\hline Article & Number \\
\hline 1-Way tips for syringes & 30 \\
\hline Load Index $(\mathbf{C})$ & $\mathbf{3 0}$ \\
\hline
\end{tabular}

J. Energy Demand of the Devices/Apparatus

\begin{tabular}{cc}
\hline Component & Energy Demand (kWh) \\
\hline AE-Spectrometer & 15.6 \\
Computer & 4.8 \\
\hline Load Index (E) & $\mathbf{2 0 . 4}$ \\
\hline
\end{tabular}

K. Weight of the Devices/Apparatus

\begin{tabular}{cc}
\hline Component & Mass (kg) \\
\hline AE- spectrometer & 90 \\
\hline Computer & 21 \\
\hline Factor 'Weight' & $\mathbf{1 1 1}$ \\
\hline
\end{tabular}

L. Lifespan of Devices/Apparatus

\begin{tabular}{cc}
\hline Use & Potentiation Factor \\
\hline Over 10 years & 1.1 \\
\hline
\end{tabular}

M. Sustainability Code \& Load Index Calculation

\begin{tabular}{cc}
\hline Substance & \\
\hline Basic Hazard Potential & 5 \\
\hline Extended Hazard Potential & 5 \\
\hline Hazard Number & 25 \\
\hline Potentiation Factor 'Quantity' & 15 \\
\hline Potentiation Factor 'Pictograms' & 1.1 \\
\hline Potentiation Factor 'Recycling/Disposal (R/D)' & 1.2 \\
\hline Load Index (M) & $\mathbf{5 0}$ \\
\hline Consumables & \\
\hline Load Index (C) & $\mathbf{3 0}$ \\
\hline & \\
\hline Energy Demand & $\mathbf{2 0}$ \\
\hline Load Index (E) \\
\hline Devices/Apparatus \\
\hline Factor 'Weight' \\
\hline Potentiation Factor 'Lifespan \\
\hline Load Index (D/A) \\
\hline
\end{tabular}

The Sustainability Code of the AES experiment was: M-50-C-30-E-20-DA-122, while the Load Index was 70.

\section{Potential Weaknesses of the Assessments}

While the suggested approach is well-suited for semi-quantification of the environmental impact of analytical processes in practical training, there are potential limitations.

One limitation is that only the most 'hazardous' material in a practical experiment is used for weighting. In our study this was done for the sake of simplification. A further limitation is the restriction of the quantities used for the weighting factors to a maximum of $1000 \mathrm{~g}$. In addition, classification is complicated by the fact that the various materials are mostly used independently or sequentially. In a conventional arrangement one would expect the respective final concentrations of all the ingredients of a mixture to be calculated before a GHS classification was made for the mixture. However, this only applies to true mixtures.

\subsection{Reasons for Weighting Only One Component}

In laboratory experiments, one substance is generally dominant in terms of quantity, while use of the other components is almost negligible. The use of the simplified calculation described above and inclusion of only one component in the weighting is therefore justified.

For the calculation of all Load Indices in this study, the method of weighting individual components was preferred, since this method does not require special software, which is sometimes expensive, and the values supplied for semi-quantitative weighting were deemed acceptable. It should be noted that the classification of mixtures which contain liquids and gases (e.g. GC, AAS, AES) would not be feasible.

If, in exceptional cases, no component was quantitatively dominant, the weighting options described below were used as an alternative.

When several substances with similar mass ratios and the same markings (hazards) were used, the corresponding amounts were added and weighted as one component.

When several substances in similar mass ratios but with different labeling were used in the experiment, weighting on the one-component basis had weaknesses, because the other components were not considered. Inserting the pictogram indicator in the weighting was an attempt to take all hazards into account. Which weighting method is more appropriate for which mass ratio could be the subject of further study.

\subsection{An Ideal Analytical Method: Conductometry}

Conductometry was chosen as an ideal analytical method in terms of sustainability because it was found to best fulfill the criteria of sustainable analysis (see section 4). 


\section{Analytical Problem}

Determination of total hardness and carbonate hardness by conductometric titration

\section{A. Substances Used}

\begin{tabular}{cc}
\hline Substance & CAS Number \\
\hline EDTA, $\mathrm{Na}_{2}$ & $6381-92-6$ \\
\hline Ethylenediaminetetraacetic acid & \\
\hline
\end{tabular}

B. Devices/Apparatus Used

\begin{tabular}{c}
\hline Main Devices \\
\hline Conductivity meter \\
\hline Computer \\
\hline
\end{tabular}

C. Hazardousness of the Substances Used

\begin{tabular}{ccc}
\hline Subtance & Hazard & (BHP) \\
\hline & GHS07 & \\
EDTA, Na 2 & H315 & 2 \\
& H319 & 7 \\
& H335 & 4 \\
\hline
\end{tabular}

D. Calculation of the Hazard Potentials

\begin{tabular}{ccccc}
\hline HC & BHP & EHP & Hazard Number & Substance \\
\hline GHS02 & 4 & 6 & & \\
\hline GHS03 & 5 & - & & \\
\hline GHS04 & 2 & - & & \\
\hline GHS05 & 12 & - & & \\
\hline GHS06 & 16 & 7 & & EDTA \\
\hline GHS07 & 6 & 7 & 42 & \\
\hline GHS08 & 8 & 9 & 72 & \\
\hline GHS09 & 3 & - & & \\
\hline
\end{tabular}

HC: Hazard Class; BHP: Basic Hazard Potential; EHP: Extended Hazard Potential

E. Hazard Potentials

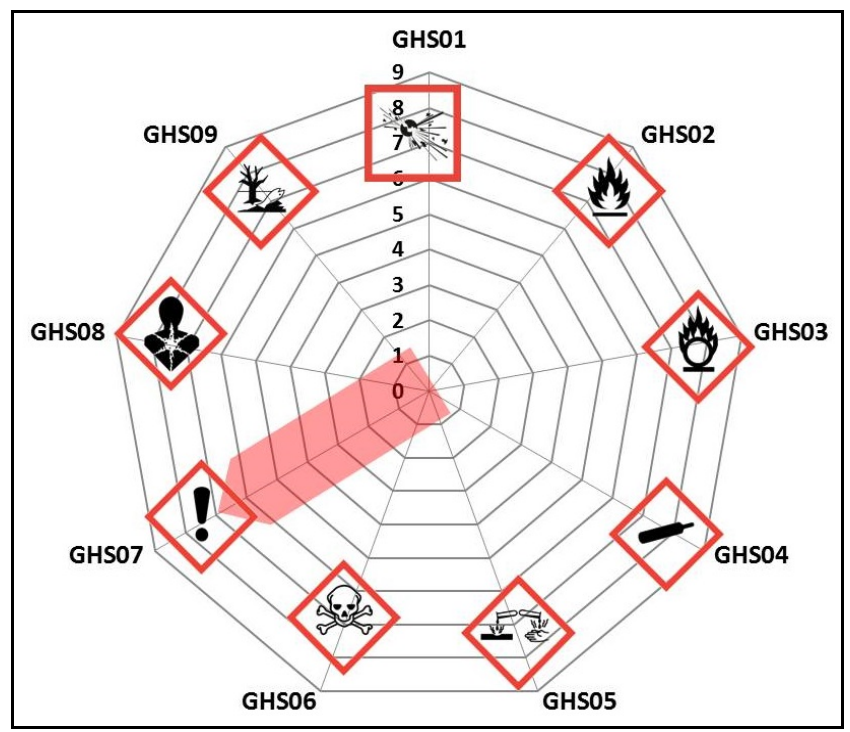

Figure 5. Qualitative Representation of the Substances Used in the Conductometry Experiment
F. Substances Used (Absolute Quantities)

\begin{tabular}{ccc}
\hline Substance & Quantity & Potentiation Factor \\
\hline EDTA, $\mathrm{Na}_{2}$ & $2 \mathrm{~g}^{*}$ & 2 \\
\hline
\end{tabular}

* ca. 35-40 determinations

G. Number of "Hazards"

\begin{tabular}{cc}
\hline Number of Pictograms & Potentiation Factor \\
\hline 1 & 1.0 \\
\hline
\end{tabular}

H. Recycling/Disposal

\begin{tabular}{cc}
\hline Type & Potentiation Factor \\
\hline Neutralization & 1.1 \\
\hline
\end{tabular}

I. Consumables

No further materials are necessary.

J. Energy Demand for Devices/Apparatus

\begin{tabular}{cc}
\hline Component & Energy Demand (kWh) \\
\hline Conductivity meter & 0.24 \\
\hline Computer & 4.8 \\
\hline Load Index (E) & 5 \\
\hline
\end{tabular}

K. Weight of Devices/Apparatus

\begin{tabular}{cc}
\hline Component & Mass (kg) \\
\hline Conductivity meter & 3 \\
\hline Computer & 21 \\
\hline Factor 'Weight' & 91 \\
\hline
\end{tabular}

L. Lifespan of Devices/Apparatus

\begin{tabular}{cc}
\hline Use & Potentiation Factor \\
\hline Over 10 years & 1.1
\end{tabular}

Over 10 years

1.1

M. Calculation of Sustainability Code \& Load Index for the Conductometry Experiment

\begin{tabular}{cc}
\hline Substances & \\
\hline Basic Hazard Potential & 6 \\
\hline Extended Hazard Potential & 7 \\
\hline Hazard Number & 42 \\
\hline Potentiation Factor 'Quantity' & 2 \\
\hline Potentiation Factor 'Pictograms' & 1 \\
\hline Potentiation Factor 'Recycling/Disposal (R/D)' & 1.1 \\
\hline Load Index (S) & $\mathbf{5 6 4}$ \\
\hline Consumables & \\
\hline Load Index (C) & \\
\hline Energy Demand & \\
\hline Load Index (E) & $\mathbf{5}$ \\
\hline & 24 \\
\hline Devices/Apparatus & 1.1 \\
\hline Factor 'Weight' \\
\hline Potentiation Factor 'Lifespan' \\
\hline Load Index (D/A)
\end{tabular}


The Sustainability Code of the Conductometry experiment was: S-9-C-0-E-5-D/A-26, while the Load Index was $\mathbf{1 4 .}$

\subsection{Comparison of the Analytical Methods}

The experiments assessed are presented for comparison in Figure 5 (Load Index) and in Tables 6-9 (Sustainability Code), in which the contribution by materials and energy to the Load Index is shown. It can be seen that the direct methods, such as UV-VIS, Raman and FTIR, require almost no materials, while most experiments require small amounts.
The maximum Load Index varies in indirect methods from about 50 for polarography to 600 for HPLC. This range from 0 to 600 allows the methods to be subjectively classified into three categories:

\section{Load Index $<20$ 'Ideal' method of analysis}

II. Load index 20-200 Sustainable method of analysis

III. Load index from 200 Method of analysis with potential for optimization

In Figures 6-8 the Sustainability Codes of the experiments carried out are grouped into chromatographic, optical and electrochemical methods.

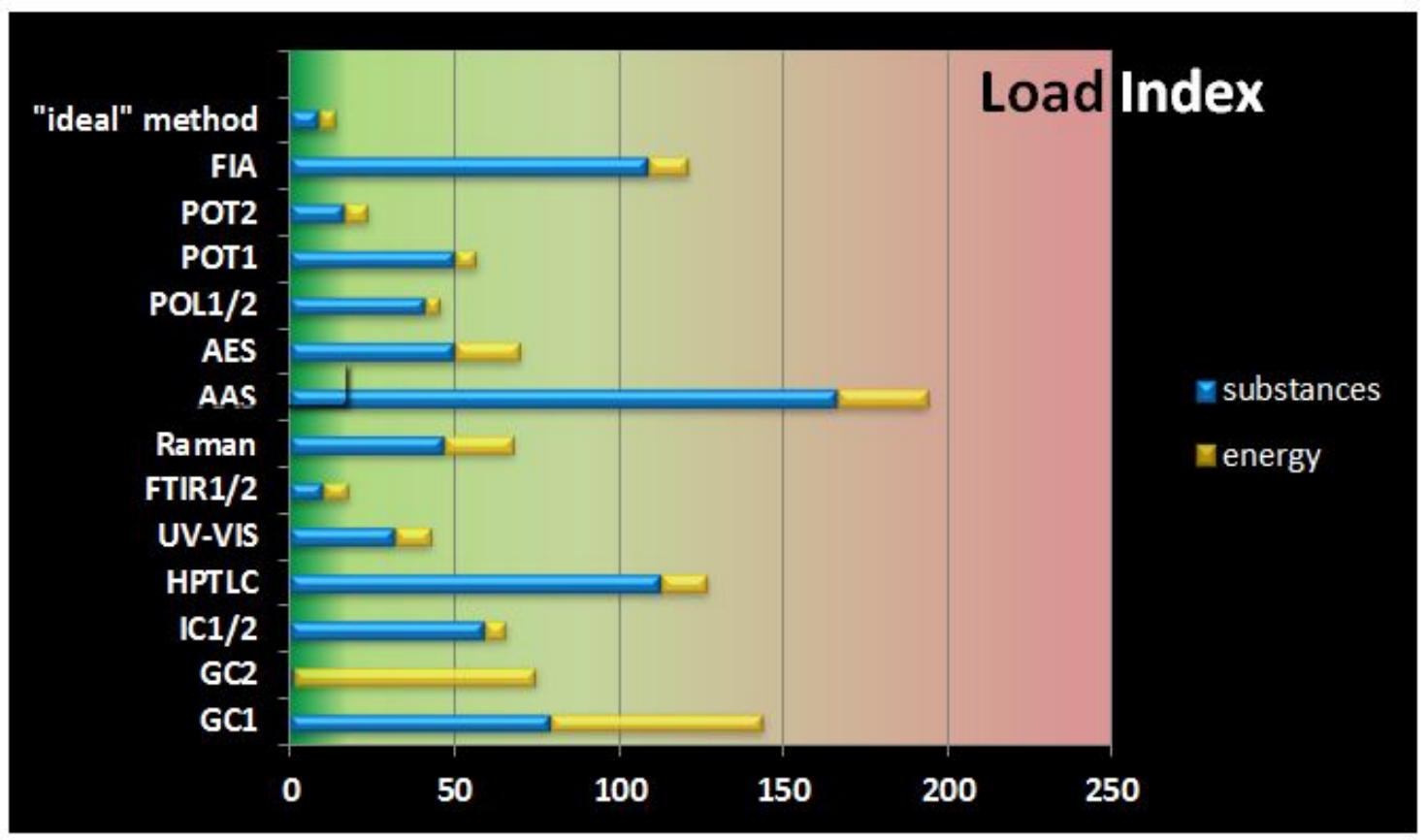

Figure 6. Diagram of the Load Indices Compared to Each Other (Not Including the HPLC Method) 


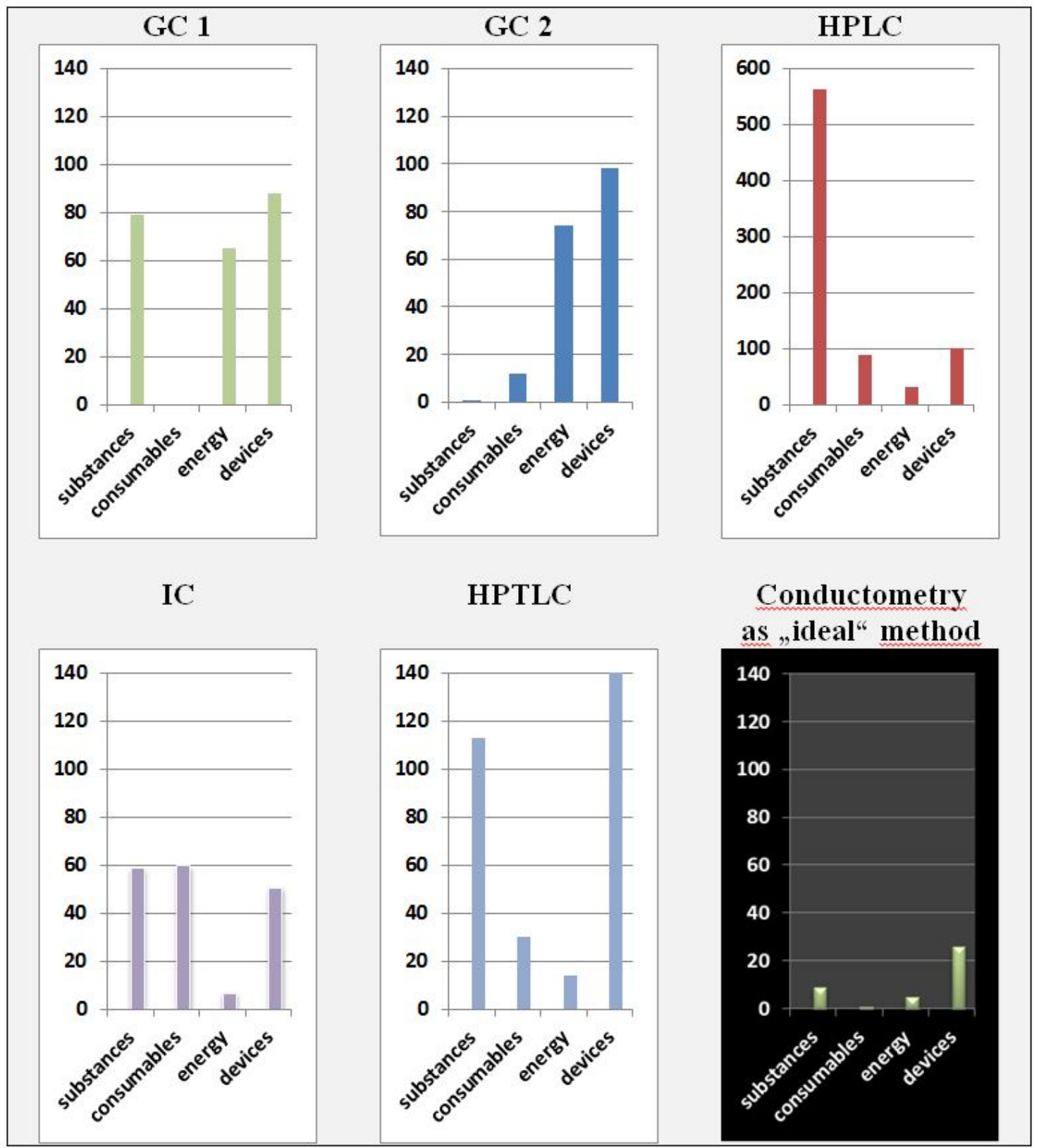

Figure 7. Summary of the Sustainability Codes for the Chromotographic Methods. The most sustainable method, conductometry, is shown for comparison. 


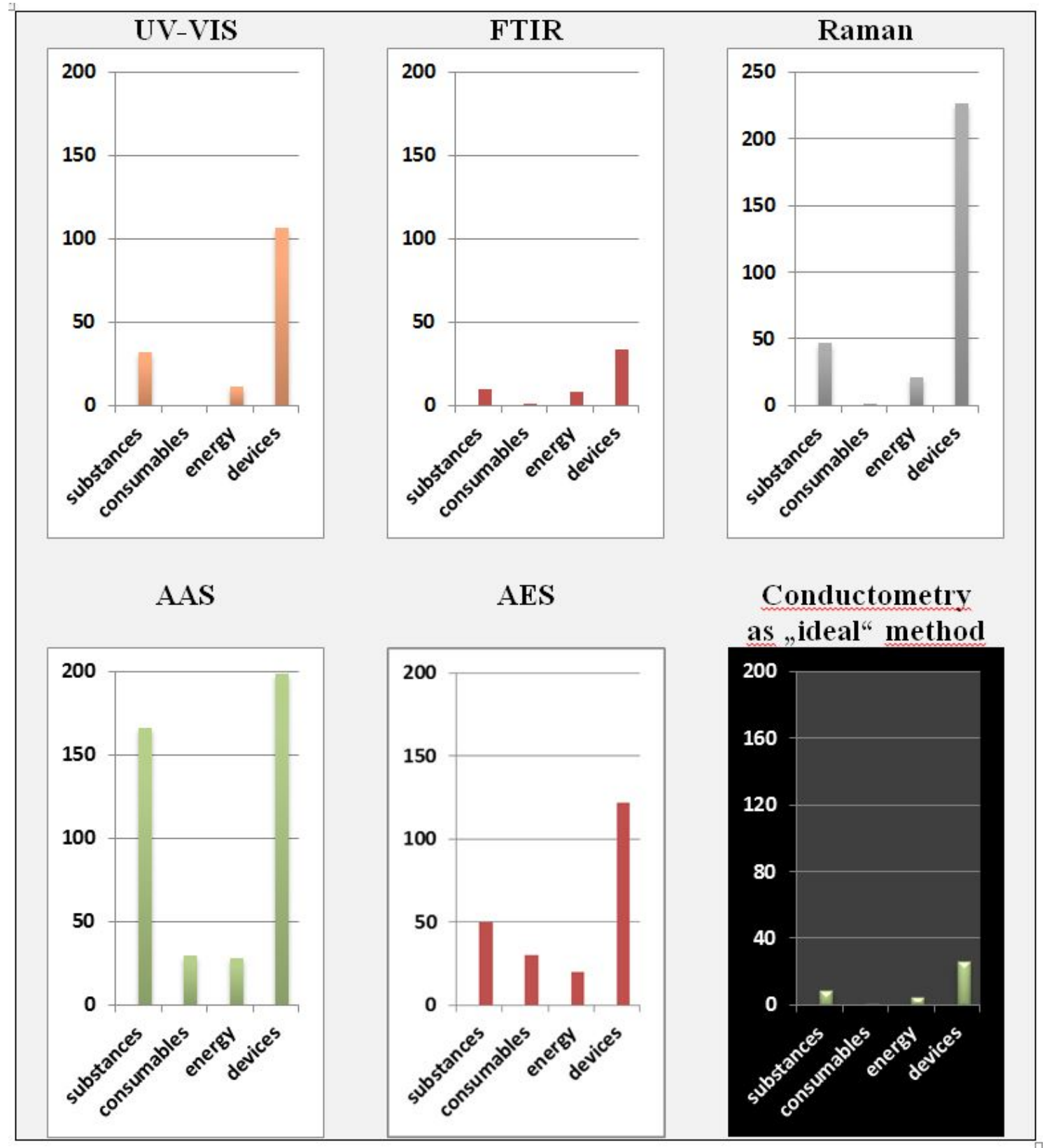

Figure 8. Summary of the Sustainability Codes for the Spectroscopic/ Spectrometric Methods. The most sustainable method, conductometry, is shown for comparison. 


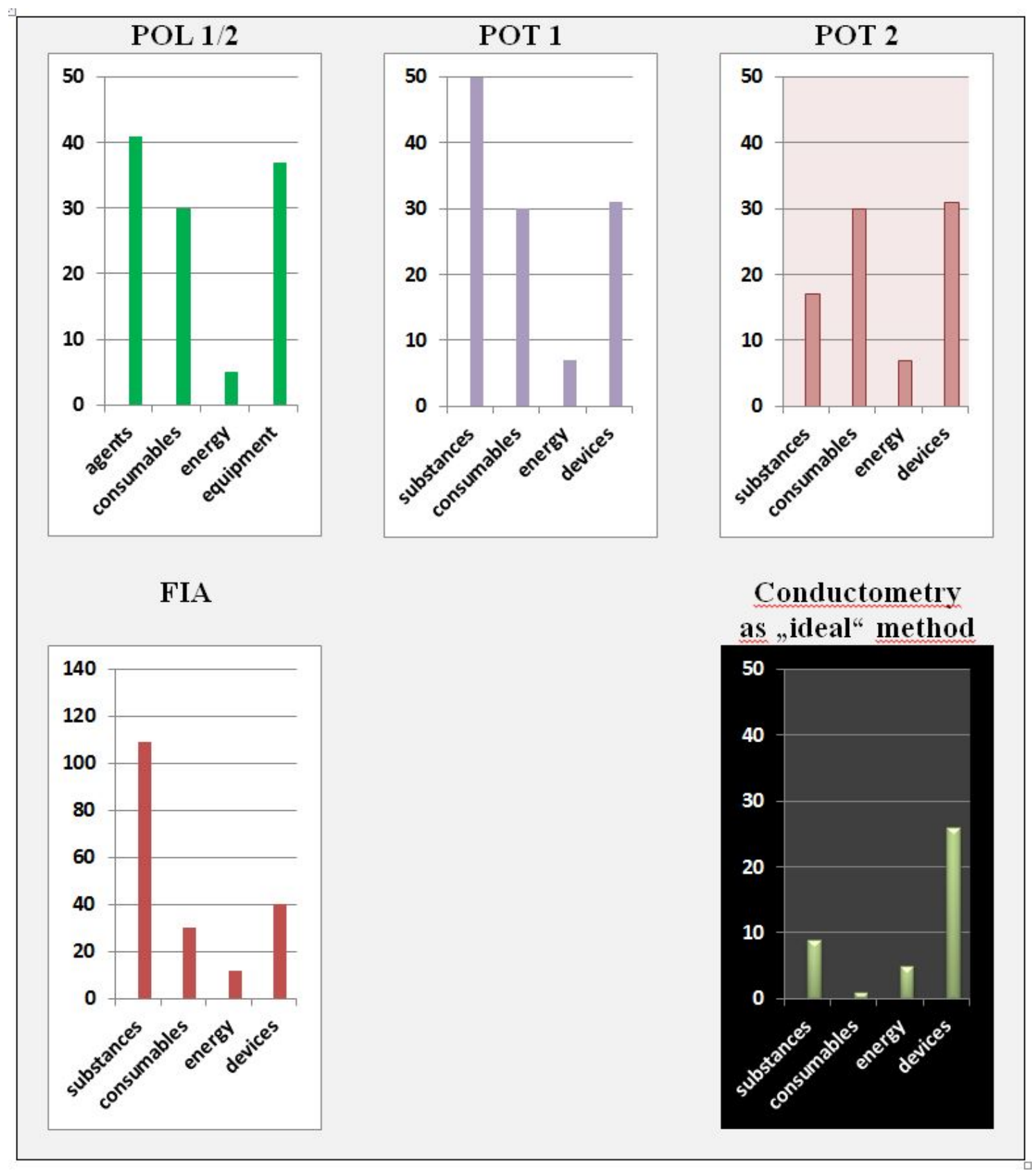

Figure 9. Summary of the Sustainability Codes for the Electrochemical Methods including FIA. The most sustainable method, conductometry, is shown for comparison.

On the basis of our subjective classification the electroanalytical methods were rated as 'ideal', while UV-VIS, FTIR, Raman and AES were classified as 'sustainable' methods. The AAS and AES methods were considered to have 'potential for optimization', together with the HPLC experiments, which have the highest Load Index of 600 .

\subsection{Optimization Approaches}

Substance-based

After weighting, optimization approaches were sought which would above all reduce the use of substances and energy consumption. Implementation of possible improvements was planned for the Fall Semester 2015, if feasible.

The targeted optimization strategy was focused, as stated above, on a general reduction in, or at least substitution of, hazardous substances.

\section{Device-related Energy Savings}

In addition to the reduction of environmental impact achieved by reducing the amounts of substances used in the experiments, energy saving is another important way to increase the sustainability of practical training. In contrast to the energy consumption of a human household (see also 8.7.1), in which saving potential is certainly present, the consumption of a given instrument is preset and cannot be 
influenced during a measurement. For this reason, only the service life can be optimized, by ensuring that devices are consistently turned off when not in use.

It should again be mentioned that the aim of this study was not to replace existing apparatus or instruments or to find alternative methods, but to achieve optimization in terms of sustainability while using existing equipment. Purchase of new energy-saving equipment could only be justified if the energy and resources required for their production were also taken into account.

\section{General Optimization Strategies}

The following general measures also contribute to energy saving, although they are not directly connected to analytical chemistry:

- Reduction of ventilation in the laboratory and in areas with high ventilation demand

- Limitation of heating in the laboratories

- Switching off lights and electrical equipment when not in use.

Computers and related equipment such as monitors etc. consume a great deal of power and should be switched off overnight or switched into standby mode for shorter periods of inactivity, e.g. when the user is taking part in meetings or seminars.

Energy efficiency should be used as a selection criterion when purchasing new lab equipment, although in many cases the intrinsic power consumption of an apparatus is fixed. For example, the total energy demand of a gas chromatograph in 1990 was $2.4 \mathrm{~kW}$, whereas that of successor models had risen to $2.7 \mathrm{~kW}$ by 2014 .

\subsection{The Sustainability Load Index as a Quality Feature}

In addition to practical laboratory courses in analytical chemistry, further compulsory traditional practical courses which form part of university curricula, could be evaluated and optimized with respect to sustainability using the method presented here. Such courses include, for example, basic chemistry, industrial chemistry, physical chemistry or bioanalysis.

Sustainability represents added value, not only in the academic context, but also for companies, with key stakeholders paying more attention to business's environmental impact. Ever-increasing awareness of environmental issues is leading to a growing demand for products that meet sustainable production criteria. Sustainability clearly has power in the marketplace today if used appropriately, providing a means for differentiation or giving greater reason to believe in a product's benefits.

The load index described in this paper could be an approach towards facilitating systematic quantification and comparison of sustainability, not only for analytical methods, but also for entire processes and products.

\section{Conclusions}

The aim of this study was to develop a comprehensive method, tailored to analytics, for assessing the sustainability of different analytical methods in practical laboratory training at a university. It should be noted that the safety of the students played a central role in this assessment. We are aware of our responsibility to place particular emphasis on sustainability regarding health and safety, as well as on environmental sustainability.

The collection of data for assessing the various analytical tests was carried out during the spring semester 2015. The results were semi-quantitatively represented, either numerically, graphically or qualitatively as a spider diagram. Certain aspects were not taken into account when assessing the substances and devices/apparatus employed. For example, origin, resource consumption, energy consumption and the resulting emissions during the production and disposal of substances or devices were not considered in the assessment. In addition, the price of a device or material was not used as a sustainability factor, since this reflects the marketing strategy of the respective companies rather than the resources used in production.

The single component method was used for the calculation of the Load Index 'Substances', as it does not require expensive software. The resulting values were deemed acceptable for semi-quantitative assessment.

It has been demonstrated that a certain value can be assigned to various aspects of sustainability in the laboratory training sequence under study, to make different methods more comparable. In addition to direct methods, such as Raman and FTIR spectroscopy, most of the experiments rated were characterized by low use of chemicals and negligible chemical waste. An exception was HPLC, in which high amounts of methanol are formed as the main waste product. It was also found that the materials used have little potential danger, either for health or the environment. Apart from the Atom Absorption Spectrometry experiment, most experiments required no sample preparation.

An 'ideal' analytical method would be for example conductometry, for which a relatively low pollution index of 14 was calculated. When all the experiments are considered, a classification into three groups can be made, based on the Load Indices determined:

A) 'Ideal' analytical methods with the lowest Load Indices of less than 20, which include electrochemical procedures such as polarography and potentiometry, because the equipment used is very light $(10 \mathrm{~kg})$ and needs very little energy (ca. $5 \mathrm{~kW} / \mathrm{h}$ ).

B) Spectroscopic and spectrometric methods (UV-VIS, FTIR, Raman, AES), which have Load Indices between 20 and 70 and were classified as sustainable methods.

C) Methods with a Load Index of over 200, which were classified as 'analytical methods with optimization potential.' 
These include the AAS method, which has a higher Load Index (200) than the related method AES, as a result of high gas consumption. The HPLC experiments were also put into this category because they had the highest Load Index of 600 . With the improvements in solvent consumption already implemented, eluent quantity per analysis was reduced by a factor of 3. Further possible optimization potential was detected and it is planned to replace heavy metals in the ICBC's UV-VIS spectroscopy, miniaturize the FIA, and replace helium with hydrogen in the GC. In the polarography method, however, substitution of mercury does not seem possible if the experimental set-up is to be maintained in its current state. In summary, the measures above can be expected to achieve maximum optimization potential regarding the use of chemicals as well as other substantial improvements.

With regard to energy demand, the use of devices/apparatus cannot be changed and therefore allows no savings potential. On the other hand, energy savings can be achieved in the lifetime of the equipment before and after an analysis, when students are informed accordingly and the devices are turned off consistently when not in use, or at least switched to standby mode.

In conclusion, it can be stated that the Practical Training Course in instrumental analysis at the Institute of Chemistry and Biological Chemistry (ICBC) at the ZHAW has been optimized in terms of sustainability. The assessment method proposed here is transparent and easy to understand and its semi-quantitative weighting procedure can be used for various analytical methods in university practical training and in laboratory practice. The assessment criteria can be modified at the user's discretion and are therefore adaptable to particular conditions. In summary, a flexible, easy and fast tool is now available to assess the "greenness" profile of an analytical method and identify optimization potential with respect to sustainability. Furthermore, this assessment tool could be used to condense information about holistic indicators and factors into a sustainability load index, which would facilitate their objective comparison and thus serve as a quality feature.

\section{Acknowledgements}

Thanks are due to Dr. Markus Zingg (ZHAW) for his valuable help in the assignment of the various Potentiation Factors.

We are particularly indebted to Dr. Sebastian Opitz (ZHAW) for the time he took to thoroughly check the manuscript.

We are also grateful to Dr. Alexia Glöss and Dr. Marco Wellinger (ZHAW) for their help in proofreading.

Our thanks also go to Nick Bell and Prof. Dr. Maggi Lussi Bell (Language Services, ZHAW) for checking the document.

\section{REFERENCES}

[1] P. Levi, The Periodic Table, translated by R. Rosenthal, Michael Joseph Ltd, London, October 1985.

[2] P. T. Anastas, I. J. Levi, K. E. Parent, Green Chemistry Education, Changing the Course of Chemistry. ACS Symposium Series 1011, 2009.

[3] J. A. Haack et al., J. Chem. Educ., Vol. 90, 515-516, 2013.

[4] T. Collins, Essays on Science and Society: Toward Sustainable Chemistry. Science, 291, 48-49, 2001.

[5] T. Collins, The Importance of Sustainability Ethics, Toxicity and Ecotoxicity in Chemical Education and Research. Green Chem., G51-G52, 2003.

[6] L. H. Keith, L. U. Gron, J. L. Young, Green Analytical Methodologies. Chem. Rev., 107, 2695-2708, 2007.

[7] National Environmental Methods Index. http://www.nemi.gov (accessed October 15, 2015).

[8] P. T. Anastas, J. C. Warner. Green Chemistry: Theory and Practice. New York: Oxford University Press, 1998.

[9] P. J. Dunn, S. Galvin and K. Hettenbach, Green Chem, Vol. 6, 43, 2004.

[10] D. J. C. Constable, A. D. Curzon and V. L. Cunningham Green Chem., Vol. 4, 451-527, 2002.

[11] L. J. Tucker, Org. Process Res. Dev., Vol. 14 (2), 328-331, 2010.

[12] R. Wojcieszak, F. Santarelli, S. Paul, F. Dumeignil, F. Cavani and R. V. Gonçalves, Sustainable Chemical Processes 2015, 3:9 (30 June 2015).

[13] B. M. Trost, Angew. Chem., Int. Ed. Engl. Vol. 34, 259, 1995.

[14] D. J. C. Constable, A. D. Curzon, D. N. Mortimer and V. L. Cunningham Green Chem., Vol. 3, 1, 2001.

[15] R. A. Sheldon, Chem. Ind. (London), Vol. 23, 903, 1992.

[16] K. Van Aken, L. Strekowski and L. Patiny, Beilstein J. Org. Chem., Vol. 2, 3, 2006.

[17] M. de la Guardia, S. Armenta. Green Analytical Chemistry: Theory and Practice. Amsterdam, The Netherlands: Elsevier, 2010.

[18] M. de la Guardia, S. Garrigues. Challanges in Green Analytical Chemistry. Cambridge, UK: Royal Society of Chemistry, 2011.

[19] M. Koel, M. Kalijurand. Green Analytical Chemistry. London, UK: Royal Society of Chemistry, 2010.

[20] M. Tobiszewski et al. Trends Anal. Chem., Vol. 28, 943, 2009.

[21] W. Wardencki et al., Polish J. Environ. Stud., Vol. 14, 389, 2005.

[22] M. Tobiszewski et al., Chem. Soc. Rev., Vol. 39, 2869, 2010.

[23] C. Bendicho, I. Lavilla, F. Pena and M. Costas, Challenges in Green Analytical Chemistry, RSC Green Chemistry No 13, ISBN: 978-1-84973-132-4, 63-106, 2011. 
[24] J. L. Young and D. E. Raynie, Challenges in Green Analytical Chemistry, RSC Green Chemistry No 13, ISBN: 978-1-84973-132-4, 44-62, 2011.

[25] S. Garrigues and M. de la Guardia, Challenges in Green Analytical Chemistry, RSC Green Chemistry No 13, ISBN: 978-1-84973-132-4, 13-43, 2011.

[26] M. Pena-Abaurrea and L. Ramos, Challenges in Green Analytical Chemistry, RSC Green Chemistry No 13, ISBN: 978-1-84973-132-4, 107-143, 2011

[27] M. de la Guardia and S. Garrigues, Handbook of Green Analytical Chemistry. John Wiley \& Sons, Ltd., Publication, 2011.
[28] D. Mangelings and Y.V. Heyden, J. Sep. Sci, Vol. 31, 1252-1273, 2008.

[29] K. W. Phinney, Anal. Bioanal. Chem., Vol. 382, 639-645, 2005.

[30] A. Rocco and S. Fanali, J. Sep. Sci, Vol. 32, 1696-1703, 2009.

[31] G. D'Orazio, S. Fanali, K. Lomsadze and B. Chankvedadze, J. Chromatogr. B, Vol. 875, 296-303, 2008.

[32] M. Koel and M. Kaljurand, Pure Appl. Chem., Vol. 78, 1993-2002, 2006.

[33] A. Gałuszka, P. Konieczka, Z. M. Migaszewski and J. Namiésnik, Trends Anal. Chem., Vol. 27, 497, 2008. 